\title{
MASTIODITE MASQUEE: A PROPOS DE DEUX OBSERVATIONS
}

\author{
A. ABDELLAOUI, M. BELCADHI, R. MANI, S. KADI, I. ZEGLAOUI, \\ M. BEN ALI, M. ABDELKEFI, K. BOUZOUITA. \\ SERVICE D'ORL ET DE CHIRURGIE CERVICOFACIALE - \\ HOPITAL UNIVERSITAIRE FARHAT HACHED - SOUSSE.
}

\begin{abstract}
La mastoïdite masquée (MM) se définit comme un état inflammatoire latent de la muqueuse et de l'os mastoïdien avec un tympan normal. Elle est révélée par une complication généralement endocrânienne. Le traitement est une mastoïdectomie associée à une antibiothérapie intraveineuse.

Nous rapportons deux observations de mastoïdites masquées dont le diagnostic a été évoqué par l'examen tomodensitométrique qui a rattaché les complications endocrâniennes à leur origine mastoïdienne.

Le but de notre travail est de discuter les circonstances diagnostiques, les investigations paracliniques et le traitement des mastoïdites masquées.
\end{abstract}

Mots-clés : mastoïdite masquée, complications endocrâniennes, mastoïdectomie.

\footnotetext{
Masked mastoiditis is defined as a subclinical infectious inflammatory process of the mucosal lining and bony structures of the mastoid air cells with an intact tympanic membrane.

It is characterized by the existence of endocranial complications, which often reveal the disease. The treatment of masked mastoiditis includes antimicrobial therapy and mastoidectomy.

We report two cases of masked mastoiditis. The diagnosis was evoked by computed tomography scan; witch shown the link between the endocranial complications and mastoid origin.

The aim of our study is to discuss diagnostic circumstances, clinic characteristics and management of masked mastoiditis.
}

Keywords : Masked mastoiditis, endocranial complications, mastoidectomy.

\section{INTRODUCTION}

La mastoïdite est une atteinte infectieuse des cavités mastoïdiennes associée à des lésions destructives de l'os mastoïdien réalisant une ostéite. Les mastoïdites masquées (MM) sont celles où le tympan reste normal. Ce sont des formes particulièrement trompeuses où le diagnostic n'est fait que devant l'apparition de complications, en particulier endocrâniennes. L'imagerie prend alors tout son intérêt puisque c'est elle qui rapporte la localisation endocrânienne à son étiologie mastoïdienne. Le traitement associe une antibiothérapie par voie intraveineuse bien adaptée et une mastoïdectomie. L'objectif de notre travail est de décrire les aspects cliniques et les examens paracliniques de cette affection et d'en discuter la prise en charge thérapeutique.

\section{MATÉRIEL ET MÉTHODES Observation 1:}

JJB, homme âgé de 40 ans, ayant comme antécédent une thrombophlébite superficielle de la jambe gauche traitée médicalement 4 ans auparavant.
Le patient a été hospitalisé au service de Médecine interne (CHU Farhat Hached de Sousse), pour une fièvre à $39^{\circ} \mathrm{C}$, des céphalées, des cervicalgies et une otalgie droite évoluant depuis 10 jours. Le bilan infectieux comportant des hémocultures, une radiographie du thorax et un ECBU était négatif.

Une tomodensitométrie cérébrale a été demandée et a objectivé une thrombophlébite du sinus latéral droit associée à une lyse osseuse du rocher du même côté (Fig. 1). L'examen otologique a trouvé du côté droit : une région rétro-auriculaire saine, un conduit auditif externe d'aspect normal et un tympan complet sans signes inflammatoires. Le reste de l'examen ORL était sans particularités. L'examen neurologique n'a pas trouvé de signes de localisation, ni de signes méningés.

L'audiométrie tonale a révélé une surdité mixte droite avec un seuil moyen à $80 \mathrm{~dB}$ et un Rinne moyen à $30 \mathrm{~dB}$. Une échographie doppler des vaisseaux du cou a montré une thrombose de la veine jugulaire interne droite. Un dosage des facteurs de coagulation a été pratiqué, il était normal. 
Le malade a été traité par une antibiothérapie par voie intraveineuse à base de céfotaxime (1gx3/j), fosfomycine (4gx3/j) et métronidazole (500mgx3/j). Après amélioration des signes généraux, une mastoïdectomie droite a été pratiquée avec une exclusion du sinus latéral, dans un délai de $48 \mathrm{~h}$. II a été constaté la présence d'une ostéite très étendue avec de nombreux séquestres osseux et l'absence de cholestéatome. Dans le même temps opératoire une cervicotomie droite avec ligature inférieure de la veine jugulaire interne a été réalisée. Les prélèvements bactériologiques per opératoires étaient revenus négatifs.

L'évolution était favorable avec disparition de la symptomatologie et retour à l'apyrexie. Le malade est régulièrement suivi à notre consultation externe et n'a pas présenté de récidive, avec un recul de 2 ans.

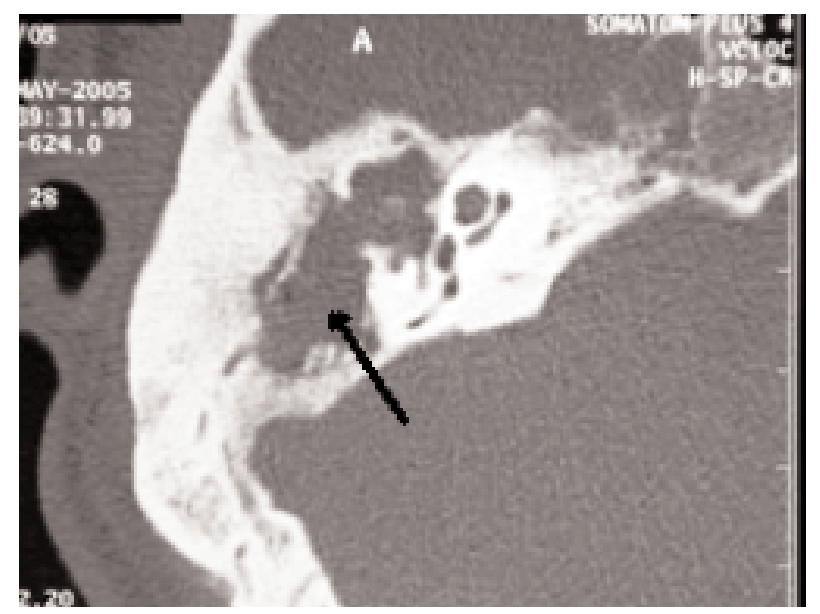

Fig. 1 : Scanner en coupe axiale montrant la lyse osseuse du rocher droit, sans abcès sous périosté associé.

\section{Observation 2 :}

MC est un enfant âgé de 13 ans, sans antécédents pathologiques notables, qui a présenté deux semaines avant son admission, une otalgie droite isolée, pour laquelle il a reçu un traitement symptomatique.

Devant l'apparition d'une fièvre non chiffrée associée à des céphalées, l'enfant a été hospitalisé. L'examen a trouvé un malade fébrile à $40^{\circ} \mathrm{C}$, conscient, sans signes de localisation neurologique ni syndrome méningé associés. L'examen otologique du côté algique a trouvé une région rétro-auriculaire saine, sans bombement ni effacement du sillon rétro-auriculaire, un conduit auditif externe libre et un tympan complet normal. L'examen de l'oreille controlatérale était normal.

Une tomodensitométrie cérébrale a été demandée et a objectivé une thrombophlébite du sinus latéral (Fig. 2) associée à un empyème temporal droit avec une lyse du rocher du même côté.

Le malade a été traité, dés son admission, par une triple antibiothérapie par voie intraveineuse : céfotaxime $(2,5 \mathrm{~g} \times 3 / \mathrm{j})$, vancomycine $(800 \mathrm{mg} \times 3 / \mathrm{j})$ et métronidazole (450mgx2/j). II a été opéré d'une mastoïdectomie droite avec exclusion du sinus latéral homolatéral, $36 \mathrm{~h}$ après le diagnostic.

L'évolution était favorable avec disparition des céphalées et de la fièvre. Le malade n'a pas gardé de séquelles et il n'a pas présenté de récidive, avec un recul de 3 ans.

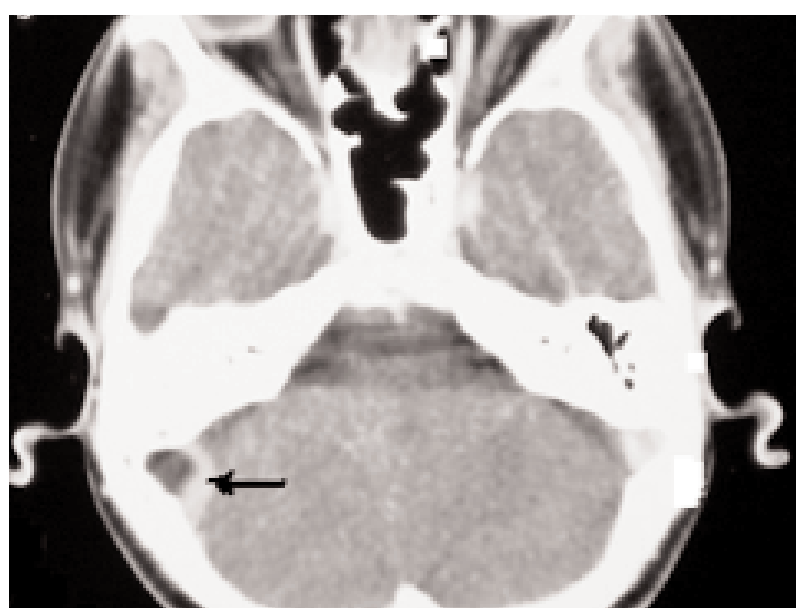

Fig. 2 : Scanner en coupe axiale avec injection de produit de contraste : thrombose du sinus latéral droit.

\section{DISCUSSION}

La mastoïdite est un processus inflammatoire de l'espace pneumatique de l'os temporal, qui complique une otite aigue ou subaigüe.

Au cours de l'ère pré-antibiotique, la mastoïdite aigue compliquait $20 \%$ des otites moyennes aigues, actuellement, ce pourcentage est de l'ordre de 0,2-2\% [1].

La mastoïdite masquée est une infection latente de la muqueuse et de l'os mastoïdien, à tympan normal, révélée par des complications.

Holt et Gates [2] se sont intéressés aux mécanismes physiopathologiques possibles de la mastoïdite masquée. Ils ont proposé le schéma suivant : I'obstruction du défilé antro-attical par une muqueuse oedématiée, polypoide ou par des formations granulomateuses rend les cellules mastoïdiennes non aérées et réalise un environnement favorable à la flore anaérobique. Cette infection anaérobique peut être insidieuse et ne pas s'exprimer par des signes évidents locaux et régionaux de la mastoïdite aigue. Le traitement antibiotique initial entraîne la guérison du processus infectieux au niveau de l'oreille moyenne. La fonction tubaire permet une aération normale de la caisse et ainsi la normalisation de l'aspect de la membrane tympanique. L'aspect otoscopique rassurant ne reflète pas la gravité du proces- 
sus ostéitique dans la mastoïde.

La maladie apparaît une ou deux semaines après une otite moyenne aigue traitée par un antibiotique à large spectre. L'otalgie est un signe pratiquement constant [3], elle a été également rapportée par nos deux malades. Les signes cliniques de la maladie sont minimaux. II peut s'agir de signes non spécifiques à type de céphalées, fièvre ou douleur rétro-auriculaire, ou bien de signes neurologiques à type de troubles visuels ou d'asymétrie faciale. Pour nos deux malades, l'examen otologique montrait une région rétro-auriculaire saine, un conduit auditif externe libre et un tympan normal. Une sensibilité à la pression de la région mastoïdienne a été observée chez un de nos patients. Le tableau clinique peut être par ailleurs évocateur d'une complication endocrânienne. Dans la série de Tovi (13 malades) [3] les complications endocrâniennes étaient présentes dans $64 \%$ des cas et dans celle de Richard (9 malades) [4], elles étaient présentes dans tous les cas. Les complications observées dans de la mastoïdite masquée sont le plus souvent endocrâniennes $[5,6]$ à type de méningites, thrombose du sinus latéral et d'abcès du cerveau, plus rarement extracrâniennes à type de labyrinthite, pétrosite et de paralysie faciale périphérique [7]. Fukuda [8] a rapporté une observation de paralysie faciale périphérique bilatérale compliquant une mastoïdite masquée bilatérale.

Sur le plan bactériologique, les agents responsables sont dans la plupart des cas des germes anaérobes [3]: peu virulents non purulents, expliquant l'absence de sécrétions purulentes dans la caisse et au niveau des cellules mastoïdiennes, d'où la négativité des cultures, chez nos deux malades. Dans la série de Tovi (13 malades) [3], l'étude bactériologique des prélèvements mastoïdiens a mis en évidence dans 4 cas des germes anaérobes. Les prélèvements des sécrétions purulentes au niveau des foyers infectieux secondaires (méningite, abcès cérébraux) ont mis en évidence, dans $30 \%$ des cas, des germes aérobes (Streptococcus pneumoniae, Escherichia coli et Protéus).

Sur le plan radiologique, l'examen tomodensitométrique montre un flou dans les espaces aériens de la mastoïde $[3,9]$. II ne montre que rarement des destructions osseuses comme dans la mastoïdite aigue. Toutefois, cet examen réalisé chez nos deux malades a montré une lyse osseuse. L'intérêt du scanner avec injection de produit de contraste intraveineux réside dans la recherche de complications endocrâniennes. Chez nos deux patients, le scanner a confirmé le diagnostic de la mastoïdite masquée en rattachant les complications endocrâniennes (thrombophlébite du sinus latéral et empyème temporal) à leur origine mastoïdienne. L'imagerie par résonance magnétique n'a pas montré sa supériorité quant au diagnostic, elle permet cependant de mieux visualiser les thrombophlébites cérébrales [11].

Selon la majorité des auteurs $[3,7,8,12]$, le traitement repose sur une antibiothérapie par voie intraveineuse devant couvrir les germes anaérobes, suivie d'une mastoïdectomie avec reperméabilisation du défilé antro-attical, dans un délai inférieur à 48 heures. Nos deux malades ont reçu une triple antibiothérapie, suivie d'une mastoïdectomie avec une exclusion du sinus latéral, respectant ce délai.

L'intervention chirurgicale permet de noter une muqueuse normale de la caisse du tympan, contrastant avec l'aspect ostéitique et inflammatoire de la mastoïde avec un défilé antro-attical complètement ou partiellement obstrué [3]. La mastoïdite masquée est également caractérisée par l'absence de sécrétions purulentes. Des bulles de gaz [3] provenant des cellules mastoïdiennes peuvent être observées au cours de l'intervention suggérant un processus infectieux anaérobique. L'analyse histologique des prélèvements mastoïdiens montre de façon constante des lésions inflammatoires chroniques de la muqueuse et de l'os avec un infiltrat fait de plasmocytes, de macrophages à cytoplasme contenant des pigments hémosidériques, des fragments osseux ostéitiques et des granulomes à cholestérine. La nécrose provoquée par la thrombose vasculaire détruit l'os, conditionne la séquestration et expose à des complications endocrâniennes. La guérison sans séquelles, au cours des mastoïdites masquées est obtenue dans deux tiers des cas $[3,4]$.

\section{CONCLUSION}

La mastoïdite masquée est une entité rare, trompeuse et grave. C'est une forme où le tympan reste normal. Généralement le diagnostic n'est fait que devant l'apparition de complications, en particulier endocrâniennes. Les signes radiologiques ne sont pas spécifiques, leur intérêt est de rapporter la complication endocrânienne à son étiologie mastoïdienne. Le traitement est basé sur une antibiothérapie par voie intraveineuse associée le plus tôt possible à une mastoïdectomie. 


\section{REFERENCES}

1- Hoppe JE, Koster S, Bootz F, Niethammer D. Acute mastoiditis. Infection. 1994; 22 : 178-82.

2- Holt G, Gates G. Masked mastoiditis. Laryngoscope. 1983; 93: 1034-7.

3- Tovi F, Gatot A, Lantsberg S. Mastoidite latente, non suppurative, masquée. Ann Otolaryngol Chir Cervicofac. 1995; 112 : 275-8.

4- Miyamoto RC, Miyamoto RT. Pediatric neurotology. Semin Pediatr Neurol. 2003; 10: 298-303.

5- Go C, Bernstein JM, de Jong AL, Sulek M, Friedman EM. Intracranial complications of acute mastoiditis. Int J Pediatr Otorhinolaryngol. 2000; 52: 143-8. 6- Kuczkowski J, Mikaszewski B. Intracranial complications of acute and chronic mastoiditis: report of two cases in children. Int J Pediatr Otorhinolaryngol. 2001; 60: 227-37.

7- Takamatsu I, Ogahara N, Fujimoto M. Two pediatric cases of facial palsy caused by masked mastoiditis, [Abstract]. Nippon Jibiinkoka Gakkai Kaiho. 1996;
99(7): 985-90.

8- Fukuda T, Sugie H, Ito M, Kikawada T. Bilateral facial palsy caused by bilateral masked mastoiditis. Pediatr Neurol. 1998; 18: 351-3.

9- Vasquez E, Castellote A. Imaging of complications of acute mastoiditis in children. Radiographics. 2003; 23: 359-72.

10- Tovi F, Gatot A. Bone scan diagnosis of masked mastoiditis. Ann Otol Rhinol Laryngol. 1992; 101: 707-9.

11- Dobben GD, Raofi B, Mafee MF. Otogenic intracranial inflammations: role of magnetic resonance imaging. Top Magn Reson Imaging. 2000; 11(2): 76-86. 12- Kuruvilla G, Job A, Mathew J. Septate fungal invasion in masked mastoiditis. J Laryngol Otol. 2006; 120(3): 250-2. 